\title{
Contents, Vol 31, 1991
}

European Neurology

Founded 1897 as 'Monatsschrift fiir Psychiatrie und Neurologie', continued 1957-1967 as 'Psychiatria et Neurologia'

Founders: C. Wernicke and Th. Ziehen.

Successors: K. Bonhoeffer (1912-1938), J. Klaesi (1939-1967),

E. Griinthal( 1953-1967)

Editor-in-Chief Editorial Board

H.E. Kaeser, Basel G. Benzi, Pavia

J. Bogousslavsky, Lausanne

E.W. Radii, Basel

H. Reichmann, Wiirzburg

Associate Editors P.J. Dyck, Rochester, Minn. P. Rondot, Paris

C. Loeb, Genova A.G. Engel, Rochester, Minn. G. Salamon, Marseille

H.P. Ludin, St. Gallen R.E. Gonsette, Melsbroek E. Satoyoshi, Tokyo

H. vander Eecken, Ghent S. Harel, Ramat Gan R. Schiffter, Berlin

W. Hartje, Aachen G. Scollo-Lavizzari, Basel

C. Hess, Bern R. Seiler, Bern

P. Huber, Bern

L. Kappos, Basel

N. Sorensen, Wiirzburg

W. Tackmann, Bonn

P. Krauseneck, Wiirzburg

Th. Landis, Zurich

P.K. Thomas, London

J. Ulrich, Basel

P.O. Lundberg, Uppsala

G.L. Mancardi, Genova

KARGER
A. Valavanis, Zurich

F. Vassella, Bern

O. Meienberg, Binningen 